\title{
Albuminuria is a marker of increasing intracranial and extracranial vascular involvement in Type 2 diabetic Chinese patients
}

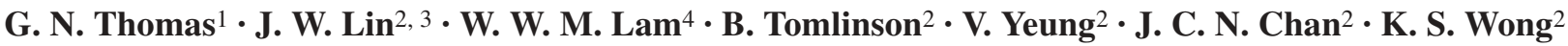 \\ ${ }^{1}$ Department of Community Medicine, 5/F Academic and Administration Block, Faculty of Medicine Building, \\ University of Hong Kong, Hong Kong, People's Republic of China \\ ${ }^{2}$ Department of Medicine and Therapeutics, The Chinese University of Hong Kong, The Prince of Wales Hospital, Hong Kong, \\ People's Republic of China \\ ${ }^{3}$ Department of Neurology, First Affiliated Hospital, Sun Yat-sen University of Medical Sciences, Guangzhou, \\ People's Republic of China \\ ${ }^{4}$ Diagnostic Radiology and Organ Imaging, The Chinese University of Hong Kong, The Prince of Wales Hospital, Hong Kong, \\ People's Republic of China
}

\begin{abstract}
Aims/hypothesis. Albuminuria has been reported to be a marker of cardiovascular risk factors and disease morbidity and mortality, but its relationship with intracerebral atherosclerotic disease is less clear. The aim of this study was to investigate the association between albuminuria and intracranial and extracranial vascular involvement in Chinese Type 2 diabetic patients.

Methods. The anthropometric and fasting biochemical measurements of 966 Type 2 diabetic patients with normoalbuminuria (55.6\%), microalbuminuria $(27.7 \%)$ or macroalbuminuria $(16.7 \%)$ were compared. The prevalence of microvascular and macrovascular disease and middle cerebral artery (MCA) stenosis, measured by transcranial Doppler ultrasound, were also compared between the groups.

Results. Albuminuria was closely associated with a range of adverse parameters, including high BP,
\end{abstract}

dyslipidaemia, smoking and adiposity (all $p<0.01$ ). The prevalence of microvascular disease (retinopathy $p<0.001$ ) and macrovascular disease (peripheral vascular disease $p=0.012$, myocardial infarction, $p=0.004$, MCA stenosis $p<0.001)$ increased significantly with increasing levels of albuminuria. Albuminuria was also found to be an independent predictor of microvascular and macrovascular disease.

Conclusions/interpretation. Albuminuria was an independent predictor of increasing levels of vascular risk factors and microvascular and macrovascular disease in this group of Type 2 diabetic patients, and a possible role for albuminuria as a marker of intracranial cerebrovascular disease should be further investigated.

Keywords BP · Cardiovascular disease - Metabolic syndrome $\cdot$ Middle cerebral artery stenosis .

Peripheral vascular disease $\cdot$ Retinopathy $\cdot$ Smoking
Received: 18 March 2004 / Accepted: 25 May 2004

Published online: 25 August 2004

(C) Springer-Verlag 2004

G. N. Thomas (-)

Department of Community Medicine,

5/F Academic and Administration Block,

Faculty of Medicine Building, University of Hong Kong,

21 Sassoon Road, Hong Kong, People's Republic of China

E-mail: gneilthomas@yahoo.co.uk

Tel.: +852-2819-9878, Fax: +852-2855-9528

Abbreviations: ABR, ankle : brachial systolic arterial pressure ratio $\cdot \mathrm{ACR}$, albumin : creatinine ratio $\cdot \mathrm{MCA}$, middle cerebral artery

\section{Introduction}

Cardiovascular disease is the major cause of morbidity and mortality in many developed and developing countries, including Hong Kong. There is a higher prevalence of stroke in Oriental populations than in Caucasian populations, where CHD generally predominates $[1,2]$. Furthermore, despite increasing cholesterol levels in Asian countries, CHD mortality rates remain three to five times lower than those in most western countries. For example, in the early 1990s, age-adjusted mortality rates (per 100,000) of 56 and 129 were reported for CHD and stroke in China, whereas the corresponding rates for the US Caucasian population were 124 and 28 , and those for the UK Caucasian population were 284 and 51 respectively 
[3]. In addition to differences in prevalence rates, the distribution of arterial atherosclerotic lesions also varies between Oriental and Caucasian populations [4, 5]. Furthermore, intracerebral haemorrhage has been reported to be two to three times more frequent in Chinese populations than in Caucasian populations, accounting for 20 to $30 \%$ of strokes [5].

Rates for CHD and stroke are subject to variation even within Chinese populations: both are higher in urban than in rural populations, and stroke rates are higher in the north of China, due to the high prevalence of hypertension [6]. These intra-ethnic differences highlight the importance of environmental and lifestyle factors in the pathogenesis of these conditions. Migration studies also support the hypothesis that changes in environmental challenges can have profound effects on the prevalence of risk factors of vascular disease [7, 8]. Based on these observations, it is likely that ethnic differences associated with cardiovascular disease are due to the interaction of external environmental factors on the different genetic backgrounds. Understanding the relative contribution of risk factors in different ethnic groups will help to clarify the potential mechanisms involved in the pathogenesis of cardiovascular disease.

The prevalence of certain conditions such as hypertension and nephropathy is higher in Chinese Type 2 diabetic patients than in Caucasian diabetic populations $[9,10]$. In Chinese diabetic patients, a random spot urine albumin : creatinine ratio (ACR) higher than $5.6 \mathrm{mg} / \mathrm{mmol}$ predicts progression to renal failure and early death [11]. Furthermore, the albuminuria associated with renal deterioration has been reported to be an index of atherosclerotic vascular disease morbidity and mortality [12, 13]. However, our understanding of the relationship between albuminuria and intracranial atherosclerotic disease is less clear.

In the current study we aimed to investigate the association between albuminuria, as determined by 24-h urinary AER, and intracranial and extracranial vascular involvement in Chinese patients with Type 2 diabetes.

\section{Subjects and methods}

The study protocol was approved by the Clinical Research Ethics Committee of the Chinese University of Hong Kong. A total of 966 Type 2 diabetic patients were recruited from the diabetes clinics at the Prince of Wales Hospital (Shatin, Hong Kong Administrative Region, People's Republic of China). All were unrelated and gave written informed consent. Subjects were of Han Chinese origin, without any known ancestors of other ethnic origin, and were living in the Hong Kong Special Administrative Region of China at the time of the study. A diagnosis of diabetes was based on a fasting plasma glucose of $\geq 7.8 \mathrm{mmol} / \mathrm{l}$ or a 2 -h OGTT (oral glucose dose $75 \mathrm{~g}$ ) plasma glucose level of $\geq 11.1 \mathrm{mmol} / 1$ [14]. Type 1 diabetic patients were defined on the basis of acute symptoms with heavy ketonuria $(>3+)$ or ketoacidosis at diagnosis or requirement for continuous insulin treatment within 1 year of diagnosis and were excluded from the study [14]. None of the screened Type 2 diabetic patients had previously suffered a stroke.

The results of seated BP, anthropometric (waist circumference and BMI) and plasma biochemical (lipid and glycaemic profiles) measurements, which were taken after an overnight fast, have previously been described in detail [10, 15]. Due to the fact that patients' medications were suspended for the morning of the assessment of trough levels, it was not possible to measure the haemodynamic parameters as continuous variables. Subjects were defined as hypertensive if their seated systolic BP was $\geq 140 \mathrm{~mm} \mathrm{Hg}$ and/or diastolic BP was $\geq 90 \mathrm{~mm}$ $\mathrm{Hg}$ on at least two occasions after 5 min rest, or if they were receiving BP-lowering medication. Patients were assessed to rule out secondary causes of hypertension and renal disease. Urine was collected over $24 \mathrm{~h}$ and the AER and ACR were determined. Urinary albumin concentration was measured by immunoturbidimetry [16]. The lowest detection limit was $2.5 \mathrm{mg} / \mathrm{l}$ and inter- and intra-assay coefficients of variation were less than $5 \%$. A midstream urine sample was collected for culture and microscopy to exclude infection. Normoalbuminuria was defined as an AER of $<20 \mu \mathrm{g} / \mathrm{min}$, microalbuminuria as an AER of $\geq 20$ to $<200 \mu \mathrm{g} / \mathrm{min}$ and macroalbuminuria as an AER of $\geq 200 \mu \mathrm{g} / \mathrm{min}$ [13]. In addition, we used an ACR of $>5.6 \mathrm{mg} / \mathrm{mmol}$ as a cut-off value as a predictor of end-stage renal disease [11]. The albuminuric status of the 966 patients with Type 2 diabetes was determined from a 24-h urine collection. The results indicated $537(55.6 \%)$ patients with normoalbuminuria, $268(27.7 \%)$ with microalbuminuria, and 161 $(16.7 \%)$ with macroalbuminuria. As age, sex and duration and age of onset of diabetes are strongly associated with diabetic complications, we also performed the analyses in a subgroup of the diabetic patients $(n=857)$ who were matched for these parameters. This subgroup comprised 475 (55.4\%) patients with normoalbuminuria, $250(29.2 \%)$ with microalbuminuria, and $132(15.4 \%)$ with macroalbuminuria. Matching was performed by excluding patients with extreme values for the parameters associated with diabetic complications.

Patients were examined by transcranial Doppler ultrasound (Nicolet EME TC 2000; Nicolet, Madison, Wis., USA); a single operator performed all evaluations. The middle cerebral artery (MCA) was studied using a standardised protocol, examining the artery by $4-\mathrm{cm}$ increments through the temporal window at 52 to $64 \mathrm{~mm}$ [17]. Due to the technical difficulty involved in differentiating vascular lesions in the terminal internal carotid artery just before the bifurcation by transcranial Doppler, we categorised these as lesions in the MCA. MCAs with a peak systolic flow velocity of $\geq 140 \mathrm{~cm} / \mathrm{s}$ were classified as occlusive [17]. In addition to the above velocity criterion, we took into account the age of the patient, the presence of turbulence or musical sound, and whether the abnormal velocity was segmental. Patients for whom insonation of the cerebral arteries was not possible through the temporal window $(13.3 \%$ of the total group) were excluded from the analyses. These patients did not exhibit any significant differences with respect to anthropometric or biochemical parameters compared with the subjects for whom insonation was performed (data not shown). The diagnostic criteria applied in our neurovascular laboratory were based on our laboratory references, which had a quality assurance programme with supplementary angiographic studies [18]. At our laboratory, we annually perform more than 1200 transcranial Doppler examinations.

Feet were examined for abnormal signs, i.e. deformities (claws toes, callus, hammer toes) and skin changes (atrophic changes such as dry skin and loss of hair, healed ulcers), as well as for distal sensory neuropathy, defined as typical symptoms (reported to the physician) of numbness or abnormal sen- 
Table 1. Characteristics of the 966 Chinese Type 2 diabetic patients categorised according to albuminuric status

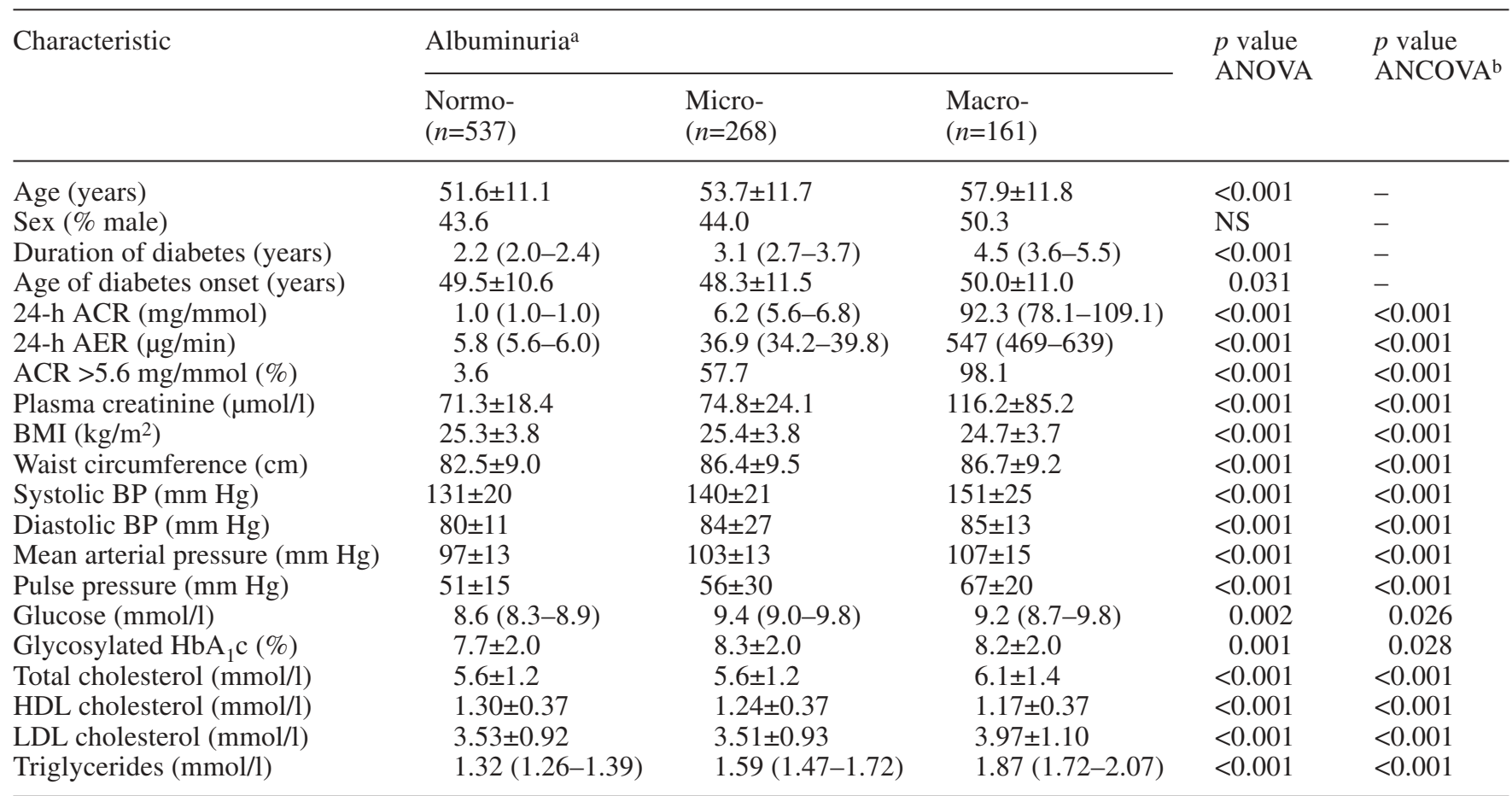

Values are means \pm SD or geometric means $(95 \%$ confidence intervals). ${ }^{a}$ Normoalbuminuria was classified as an AER of $<20 \mu \mathrm{g} / \mathrm{min}$, microalbuminuria as an AER of $\geq 20$ to $<200 \mu \mathrm{g} / \mathrm{min}$ and macroalbuminuria as an AER of $\geq 200 \mu \mathrm{g} / \mathrm{min}[13]$; ${ }^{\mathrm{b}} p$ values adjusted for age, sex and duration and age of diabetes onset. ANCOVA, analysis of covariance; NS, not significant sation in the feet accompanied by at least one physical sign or by two abnormal signs in the absence of symptoms. Physical signs included diminished ankle reflexes or sensation using a monofilament (diminished sensation over foot dorsum or sole with normal skin) or graduated tuning fork where normality was defined as $\geq 6 / 8$ or $\geq 4 / 8$ in those $<65$ or $\geq 65$ years old, respectively. The patient was considered to be at risk of developing diabetic foot syndrome if two of three of these risk factors (symmetrical sensory neuropathy, deformities or skin changes) were present. Foot pulses were examined in each patient and the ankle : brachial systolic arterial pressure ratio (ABR) was determined by Doppler examination in those with abnormal pulses. An ABR of $<0.9$ in either leg was considered suggestive of peripheral vascular disease, while a value of $<0.7$ was considered confirmatory [19]. Retinopathy was assessed by an ophthalmologist in all the patients. The fundi were examined through dilated pupils and retinopathy was considered to be present if there were one or more areas of haemorrhages, microaneurysms, cotton wool spots and/or laser coagulation scars related to diabetic retinopathy. Symptomatic peripheral vascular disease was diagnosed if claudication, gangrene or ischaemia-related amputation were present. History of coronary and cerebrovascular disease was recorded, as was the use of alcohol and tobacco products.

Statistical methods. Data from normally distributed parameters are presented as means $\pm \mathrm{SD}$, whereas skewed data were logarithmically transformed and expressed as geometric means with $95 \%$ confidence intervals. Differences in anthropometric and fasting plasma biochemical parameters between patients with increasing levels of albuminuria were examined using ANOVA and analysis of covariance to adjust for age, sex, duration of diabetes and age of diabetes onset. Dichotomous vari- ables were compared using the chi square test. A $p$ value of less than 0.05 was considered statistically significant. Sex was coded as 0 and 1 for male and female, respectively.

All 966 diabetic patients were included in the regression analyses. Stepwise multiple linear regression analysis was used to determine independent predictors of albuminuria (as determined by 24-h urinary AER). These variables included age, sex, BMI, waist circumference, systolic BP, pulse pressure, diagnosis of hypertension, glucose, triglycerides, HDL and LDL cholesterol levels and duration of diabetes.

MCA stenosis and retinopathy were chosen as examples of macrovascular and microvascular disease and were included in the forward conditional linear regression analyses to determine independent predictors of these conditions. The variables included were as described above for the stepwise multiple regression analysis and were linearly related to the dependent variable. The Statistical Package for the Social Sciences was used for the analyses (SPSS version 11.0.1, 2001; SPSS Chicago, Ill., USA).

\section{Results}

The albuminuric status of the 966 patients with Type 2 diabetes was determined from a 24-h urine collection which identified $537(55.6 \%)$ patients with normoalbuminuria, $268(27.7 \%)$ with microalbuminuria and $132(16.7 \%)$ with macroalbuminuria. Worsening albuminuria was associated with a significant increase in general (BMI) and central (waist circumference) adiposity (Table 1). Although increasing proportions of 
Table 2. Prevalence of concomitant disorders and smoking and alcohol consumption in the Chinese Type 2 diabetic patients categorised according to albuminuric status

\begin{tabular}{|c|c|c|c|c|}
\hline \multirow[t]{2}{*}{ Disorder } & \multicolumn{3}{|l|}{ Albuminuria $^{\mathrm{a}}$} & \multirow[t]{2}{*}{$p$ value } \\
\hline & $\begin{array}{l}\text { Normo- } \\
(n=537)\end{array}$ & $\begin{array}{l}\text { Micro- } \\
(n=268)\end{array}$ & $\begin{array}{l}\text { Macro- } \\
(n=161)\end{array}$ & \\
\hline MCA stenosis 1 vessel/2 vessels/combined & $6.1 / 4.5 / 10.6$ & $6.7 / 8.2 / 14.9$ & $9.8 / 15.3 / 25.1$ & $<0.001$ \\
\hline Foot affected & 2.6 & 4.5 & 12.3 & $<0.001$ \\
\hline Impotence (\% of males) & 18.7 & 20.0 & 33.8 & 0.012 \\
\hline Retinopathy & 13.2 & 32.3 & 57.7 & $<0.001$ \\
\hline Prevalence of hypertension (Rx) & $40.6(19.6)$ & $59.3(31.1)$ & $81.0(54.2)$ & $<0.001(<0.001)$ \\
\hline Prevalence of dyslipidaemia (Rx) & $44.3(3.6)$ & $49.1(4.8)$ & $68.3(8.9)$ & $<0.001(0.012)$ \\
\hline Treatment of diabetes & 72.6 & 81.8 & 86.5 & $<0.001$ \\
\hline Smoking consumption (ex/current) & $11.9 / 13.2$ & $13.4 / 17.2$ & $21.7 / 16.8$ & 0.005 \\
\hline Alcohol consumption (ex/current) & $9.5 / 8.2$ & $13.9 / 7.1$ & $18.9 / 6.9$ & NS \\
\hline
\end{tabular}

Values are $\%$. a Normoalbuminuria was classified as an AER of $<20 \mu \mathrm{g} / \mathrm{min}$, microalbuminuria as an AER of $\geq 20$ to $<200 \mu \mathrm{g} / \mathrm{min}$ and macroalbuminuria as an AER of $\geq 200 \mu \mathrm{g} / \mathrm{min}$ [13]. NS, not significant; Rx, receiving therapy

patients with microalbuminuria and macroalbuminuria received BP- and lipid-lowering therapies compared with those with normoalbuminuria (Table 2), BP and lipid (total cholesterol, LDL cholesterol and triglyceride) parameters increased significantly with increasing albumin levels, whilst HDL cholesterol levels decreased (Table 1). The changes in LDL cholesterol levels were particularly evident in the group with macroalbuminuria. The proportion of current smokers increased with increasing albuminuria; the majority of current smokers were men $(89.6 \%)$. Similar results were observed for the 857 matched diabetic patients, except for glycosylated haemoglobin levels, which did not reach significance (data not shown).

The prevalence of MCA stenosis, measured using transcranial Doppler, was twice as high in the macroalbuminuria group compared with that in the normoalbuminuria group (Fig. 1, Table 2), and the proportion of patients with stenosis in both arteries was nearly three times as high. One-quarter of the patients with macroalbuminuria had evidence of stenosis in the MCA. Concomitant increases in a number of other macrovascular conditions were also noted in this group, with significant increases observed in the prevalence of myocardial infarction and markers of peripheral vascular disease, including diabetic foot complications and impotency. Similarly, the microvascular complication, retinopathy, was significantly higher in those with macroalbuminuria, being found in over $50 \%$ of the subjects, which was 3.4 times more frequently than in the diabetic patients with normoalbuminuria.

A large number of the cardiovascular risk factors were independent predictors of 24-h urinary AER. These included systolic BP $(\beta=0.21, p<0.001)$, duration of diabetes $(\beta=0.19, \quad p<0.001)$, triglycerides $(\beta=0.10, p=0.008)$, male sex $(\beta=-0.09, p=0.008), \mathrm{LDL}$

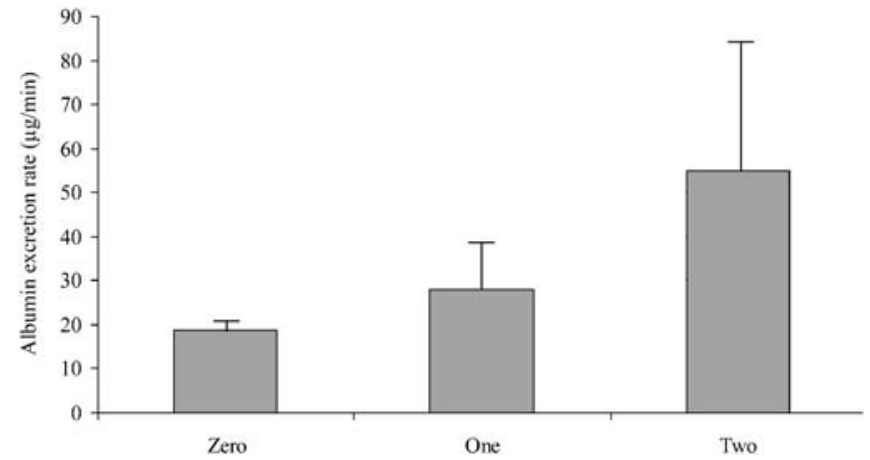

Fig. 1. Increasing 24-h urinary AER in 966 Chinese Type 2 diabetic patients with MCA stenoses in zero, one or two vessels (ANOVA, $p<0.001$, error bars represent AER standard deviation)

cholesterol $(\beta=0.11, p<0.001)$, hypertension $(\beta=0.13$, $p=0.001)$, BMI $(\beta=0.09, \quad p=0.002)$ and smoking $(\beta=0.07, p=0.033)$. These factors accounted for $21 \%$ of the variance. The following regression equation was used: 24 -h urinary $\mathrm{AER}=(0.007 \times$ systolic $\mathrm{BP})+$ $(0.24 \times$ duration of diabetes $)+(0.32 \times$ triglycerides $)-$ $(0.13 \times$ male sex $)+(0.08 \times$ LDL cholesterol $)+(0.20$ $\times$ diagnosis of hypertension $)+(0.02 \times \mathrm{BMI})+(0.07 \times$ smoking) $-0.62 ; r^{2}=0.21, F=34.8, p<0.001$.

The forward conditional linear regression analyses identified urinary AER as an independent predictor of both microvascular and macrovascular conditions. The following regression equations were used: (i) MCA stenosis $=(0.03 \times$ age $)+(0.49 \times$ AER $)-3.90$; Nagelkerke $r^{2}=0.07, \chi^{2}=32.5, p<0.001$ and (ii) retinopathy $=(-0.49 \times$ male sex $)+(1.04 \times$ AER $)+(1.63 \times$ duration of diabetes $)+(1.91 \times$ glucose $)+(0.02 \times$ pulse pressure) - 6.16; Nagelkerke $r^{2}=0.41, \chi^{2}=276.7$, $p<0.001$. 


\section{Discussion}

There is a clear relationship between ageing, sex and the duration and onset of diabetes and the development of cardiovascular and renal vascular complications $[9,10,11,12,13]$. We therefore performed analyses to adjust for these parameters in the study group as a whole and matched a subgroup of the diabetic patients with normoalbuminuria, microalbuminuria and macroalbuminuria in terms of these parameters to limit any potential confounding effect. Both adjusted analyses revealed that increasing levels of albuminuria were closely associated with increases in a number of cardiovascular risk factors, including adiposity, BP and lipid levels, with these parameters being independent predictors of 24-h AER. This supports the concept that the adverse metabolic milieu in patients with diabetes increases the risk of developing nephropathy and cardiovascular disease [10]. Albuminuria has been reported to be a marker of atherosclerotic disease, rather than directly promoting vascular disease. The concomitant presence of these risk factors is likely to promote endothelial dysfunction throughout the vasculature [20], including vessels in the glomeruli. High BP, glucose and lipid levels have all been associated with alterations in the permeability of glomerular capillaries to protein, resulting in proteinuria $[21,22]$.

Smoking has been reported to be a major risk factor for atherogenesis and vascular disease mortality [23, 24]. Reflecting smoking habits in Hong Kong general$1 y, 90 \%$ of the smokers in this study were men, and one-third of men with macroalbuminuria currently smoked whereas the corresponding figure for women in the same group was only $3 \%$. In the current study, smoking was an independent predictor and the prevalence of smoking increased with increasing albuminuria, supporting an involvement in vasculature damage. Tobacco smoke, which contains more than 4000 chemicals [25], can damage the vascular endothelium directly [26]. Furthermore, smoking can increase oxidative stress and levels of acute phase proteins [26, 27]. These parameters have been reported to be increased in the presence of microalbuminuria even in the absence of smoking, suggesting that the development of albuminuria involves an inflammatory component [28].

Type 2 diabetes is closely associated with microvascular and macrovascular disease, which are the major cause of morbidity and mortality in these patients [29]. Our study demonstrated an association between albuminuria and cardiovascular events as well as other vascular disease entities [30, 31]. The transcranial Doppler measurement of intracranial stenoses was a key measure of cerebrovascular disease in our study. A previous study performed in Hong Kong, which consecutively recruited 705 stroke patients, identified occlusive arteries in 345 patients (49\%) [17]. Of these, $37 \%$ had intracranial stenosis alone, $10 \%$ had tandem (intracranial and extracranial) lesions and 2.3\% had extracranial lesions alone [17]. Stenosis of the MCA was the most common lesion and was observed in $35.9 \%$ of the patients [17]. In the current study, we found a clear relationship between increasing levels of albuminuria and the presence of MCA stenosis, although age and albuminuria collectively accounted for only $7 \%$ of the variance. This suggests that additional parameters, not measured in this study, further contribute to the development of intracranial atherosclerotic disease. We have previously shown that an increased systolic BP, increased LDL cholesterol and a longer duration of diabetes are associated with the presence of MCA stenosis in Type 2 diabetic patients [32]. However, in contrast with the present results, this study observed lower plasma glucose levels in the group with MCA stenosis [32]. Given the chronic nature of the development of stenotic arteries, it is possible that different parameters contribute at different stages in the pathogenesis, which a cross-sectional study such as this would not be able to determine.

The conventional vascular risk factors (including albuminuria) explained a greater proportion of the variance $(41 \%)$ of the microvascular diabetic complication, retinopathy. Diabetic eye disease is the leading cause of new cases of blindness in many developed countries, for which macular oedema and proliferative retinopathy are the major causes of loss of vision [33]. In the UK Prospective Diabetes Study, tight control of BP and glycaemia effectively reduced the development of retinopathy $[34,35]$. These parameters were also independent predictors of retinopathy in the present study.

Given the close association between multiple vascular risk factors and albuminuria, it should be considered useful as an early indicator of both microvascular and macrovascular atherosclerotic disease. Microalbuminuria is also a marker of the development of renal failure [13], and we have previously reported that an ACR higher than $5.6 \mathrm{mg} / \mathrm{mmol}$ is a predictor of early progression to end-stage renal disease and subsequent mortality in Chinese Type 2 diabetic patients [11]. It is therefore necessary to develop strategies to optimise all of these risk factors, including albuminuria, to reduce the incidence of the associated devastating cardiovascular complications. However, it is important to recognise the therapeutic challenges involved in the management of Type 2 diabetes. Despite participating in a clinical trial that followed national treatment guidelines, patients in the Steno-2 study [36] did not show an improvement in glycosylated haemoglobin levels. Even in the intensive treatment group, levels were only reduced by $0.5 \%$, indicating that a significant proportion of the patients did not reach optimal glycaemic control. Data from the US Third National Health and Nutrition Examination Survey showed that between $35 \%$ and $50 \%$ of patients had very poor glycaemic control, with glycosylated haemoglobin levels 
above $8 \%$, and only $44.6 \%$ of the population had levels under 7\% [37].

In summary, albuminuria was an independent predictor of increasing levels of vascular risk factors and microvascular and macrovascular disease in this group of Type 2 diabetic patients, and a possible role for albuminuria as a marker of early cerebrovascular disease should be further investigated.

Acknowledgements. This work was supported by grants from the Hong Kong Research Grants Council (CUHK 4280/97M and CUHK 4095/00M). None of the authors are aware of any conflict of interest regarding the collection and interpretation of these data.

\section{References}

1. World Health Organization (1995) World Health Statistics Annual 1994. World Health Organization, Geneva

2. Sacco RL (1997) Risk factors, outcomes, and stroke subtypes for ischemic stroke. Neurology 49:S39-S44

3. Young RP, Thomas GN, Critchley JAJH, Tomlinson B, Woo KS, Sanderson JE (1998) Interethnic differences in coronary heart disease mortality in 25 populations: association with the angiotensin-converting enzyme DD genotype frequency. J Cardiovasc Risk 5:303-307

4. Wong KS, Huang YN, Gao S, Lam WW, Chan YL, Kay R (1998) Intracranial stenosis in Chinese patients with acute stroke. Neurology 50:812-813

5. Kay R, Woo J, Kreel L, Wong HY, Teoh T, Nicholls MG (1992) Stroke subtypes among Chinese living in Hong Kong. The Shatin Stroke Registry. Neurology 42:985-987

6. He J, Klag MJ, Wu ZG, Whelton PK (1995) Stroke in the People's Republic of China: I. Geographic variations in incidence and risk factors. Stroke 26:2222-2227

7. He J, Klag MJ, Whelton PK, Chen JY, Mo JP, Qian MC (1991) Migration, blood pressure pattern, and hypertension: the Yi Migrant Study. Am J Epidemiol 134:1085-1101

8. Robertson TL, Kato H, Gordon T et al. (1977) Epidemiologic studies of coronary heart disease and stroke in Japanese men living in Japan, Hawaii and California. Coronary heart disease risk factors in Japan and Hawaii. Am J Cardiol 39:244-249

9. Chan JCN, Cheung CK, Swaminathan R, Nicholls MG, Cockram CS (1993) Obesity, albuminuria and hypertension among Hong Kong Chinese with non-insulin-dependent diabetes mellitus (NIDDM). Postgrad Med J 69:204-210

10. Thomas GN, Critchley JAJH, Tomlinson B, Lee ZSK, Young RP, Chan JCN (2001) Albuminuria and the reninangiotensin system gene polymorphisms in Type 2 diabetic and in normoglycaemic hypertensive Chinese. Clin Nephrol $55: 7-15$

11. Chan JCN, Cheung CK, Cheung MYF, Swaminathan R, Critchley JAJH, Cockram CS (1995) Abnormal albuminuria as a predictor of mortality and renal impairment in Chinese patients with NIDDM. Diabetes Care 18:10131014

12. Mogensen CE, Poulsen PL (1994) Epidemiology of microalbuminuria in diabetes and in the background population. Curr Opin Nephrol Hypertens 3:248-256

13. Mogensen CE, Vestbo E, Poulsen PL et al. (1995) Microalbuminuria and potential confounders. A review and some observations on variability of urinary albumin excretion. Diabetes Care 18:572-581
14. World Health Organization (1997) Report of the expert committee on the diagnosis and classification of diabetes mellitus. Diabetes Care 20:1183-1197

15. Thomas GN, Tomlinson B, Chan JCN, Sanderson JE, Cockram CS, Critchley JAJH (2001) Renin-angiotensin system gene polymorphisms, blood pressure, dyslipidemia and diabetes in Hong Kong Chinese: A significant association of the ACE insertion/deletion polymorphism with type 2 diabetes. Diabetes Care 24:356-361

16. Cheung CK, Swaminathan R (1987) Rapid, economical immunoturbidimetric method for albuminuria. Clin Chem 33:204-205

17. Wong KS, Li H, Chan YL et al. (2000) Use of transcranial doppler ultrasound to predict outcome in patients with intracranial large-artery occlusive disease. Stroke 31:26412647

18. Gao S, Lam WWM, Chan YL, Liu JY, Wong KS (2002) The optimal values of flow velocity on transcranial Doppler in grading middle cerebral artery stenosis in comparison with magnetic resonance angiography. J Neuroimaging 12:213-218

19. Leng GC, Lee AJ, Fowkes FG et al. (1996) Incidence, natural history and cardiovascular events in symptomatic and asymptomatic peripheral arterial disease in the general population. Int J Epidemiol 25:1172-1181

20. Stehouwer CD, Nauta JJ, Zeldenrust GC, Hackeng WH, Donker AJ, den Ottolander GJ (1992) Urinary albumin excretion, cardiovascular disease, and endothelial dysfunction in non-insulin-dependent diabetes mellitus. Lancet 340:319323

21. Moorhead JF, Chan MK, El-Nahas M, Varghese Z (1982) Lipid nephrotoxicity in chronic progressive glomerular and tubulo-interstitial disease. Lancet 2:1309-1311

22. Nestler JE, Barlascini CO, Tetrault GA, Fratkin MJ, Clore JN, Blackard WG (1990) Increased transcapillary escape rate of albumin in non-diabetic men in response to hyperinsulinemia. Diabetes 39:1212-1217

23. U.S. Department of Health and Human Services (1990) The health benefits of smoking cessation: a report of the Surgeon General, 1990. U.S. Department of Health and Human Services, Public Health Service, Centers for Disease Control, Center for Chronic Disease Prevention and Health Promotion, Office on Smoking and Health. DHHS Publication No. (CDC) 90-8416, Rockville, MD, pp 191197

24. Lam TH, Ho SY, Hedley AJ, Mak KH, Peto R (2001) Mortality and smoking in Hong Kong: case-control study of all adult deaths in 1998. Br Med J 323:361-367

25. Taylor AE, Johnson DC, Kazemi H (1992) Environmental tobacco smoke and cardiovascular disease: a position paper from the Council on Cardiopulmonary and Critical Care, American Heart Association. Circulation 86:699-702

26. Vapaatalo H, Mervaala E (2001) Clinically important factors influencing endothelial function. Med Sci Monit 7:1075-1085

27. Lam TH, Liu LJ, Janus ED, Bourke C, Hedley AJ (1999) The relationship between fibrinogen and other coronary heart disease risk factors in a Chinese population. Atherosclerosis 143:405-413

28. Festa A, D’Agostino R, Howard G, Mykkanen L, Tracy RP, Haffner SM (2000) Inflammation and microalbuminuria in nondiabetic and type 2 diabetic subjects: The Insulin Resistance Atherosclerosis Study. Kidney Int 58:17031710

29. Chan JCN, Cockram CS (1997) Diabetes in the Chinese population and its implications for health care. Diabetes Care 20:1785-1790 
30. Diabetes Drafting Group (1985) Prevalence of small vessel and large vessel disease in diabetic patients from 14 centres. The World Health Organization Multinational Study of Vascular Disease in Diabetics. Diabetologia 28:615640

31. Ito H, Harano Y, Suzuki M et al. (1996) Risk factor analyses for macrovascular complication in nonobese NIDDM patients. Multiclinical Study for Diabetic Macroangiopathy (MSDM). Diabetes 45 [Suppl 3]:S19-S23

32. Thomas GN, Lin JW, Lam WWM et al. (2003) Middle cerebral artery stenosis in Type 2 diabetic Chinese patients is associated with conventional risk factors, but not with polymorphisms of the renin-angiotensin system genes. Cerebrovascular Diseases 16:217-223

33. Klein R, Klein BEK, Moss SE (1984) Visual impairment in diabetes. Ophthalmology 91:1-9
34. UK Prospective Diabetes Study Group (1998) Intensive blood glucose control with sulphonylureas or insulin compared with conventional treatment and risk of complications in patients with type 2 diabetes (UKPDS 33). Lancet 352:837-853

35. UK Prospective Diabetes Study Group (1998) Tight blood pressure control and risk of macrovascular and microvascular complications in type 2 diabetes (UKPDS 38). $\mathrm{Br}$ Med J 317:703-713

36. Gaede P, Vedel P, Larsen N, Jensen GV, Parving HH, Pedersen O (2003) Multifactorial intervention and cardiovascular disease in patients with type 2 diabetes. $\mathrm{N}$ Engl J Med 348:383-393

37. Harris MI, Eastman RC, Cowie CC, Flegal KM, Eberhardt MS (1999) Racial and ethnic differences in glycemic control of adults with type 2 diabetes. Diabetes Care 22:403-408 